http://jmscr.igmpublication.org/home/

ISSN (e)-2347-176x ISSN (p) 2455-0450

crossref DOI: https://dx.doi.org/10.18535/jmscr/v7i7.158

Journal Of Medical Science And Clinical Research

IGM Publication

An Official Publication of IGM Publication

\title{
Rhinosporidiosis Presenting with Stridor - A Case Series
}

\author{
Authors \\ Dr Debabrata Biswas, Dr Ishita Sen* \\ Dept of Otorhinolaryngology and Head Neck Surgery, R.G. Kar Medical College and Hospital, \\ 1, Kshudiram Bose Sarani, Kolkata-700004, India \\ *Corresponding author
}

Dr Ishita Sen

\begin{abstract}
Introduction: Rhinosporidiosis is a granulomatous disease presenting with friable polypoidal mass involving the mucous membrane mainly involving nose can present with stridor.

Case Series

Case 1, 2, 3: Nasal rhinosporidiosis with oropharyngeal and hypopharyngeal extension presented with stridor.

Case 4, 5: Laryngeal rhinosporidiosis

Conclusion: Rhinosporidiosis presenting with stridor is a rare presentation. It was managed with tracheostomy and treated by various techniques- nasal endoscopic excision, peroral excision, laryngofissure surgery followed by dapsone treatment to prevent recurrence.

Keywords: Rhinosporidiosis, Stridor, Dapsone, Laryngofissure, Tracheostomy.

Abbreviations: CT-Computed Tomography, FOL - Fibre optic Laryngoscopy.
\end{abstract}

\section{Introduction}

Rhinosporidiosis is a chronic granulomatous disease affecting the mucous membrane that usually manifests as vascular friable polyps. Rhinosporidiosis is classified as fungal disease under ICD-10. It caused by Rhinosporidium Seeberi $_{1}$. Rhinosporidiosis mainly involves the mucous membrane of nose in $78 \%$ and nasopharynx in $68 \%$, tonsils in $3 \%$ and eye in $1 \%$ of the persons with the infection ${ }_{2}$. Other structures involved are mouth, eye, skin, larynx, trachea, bronchi, genitals, rectum. It is more commonly seen in males; Male: Female - 4:1. It is most commonly found to occur in children and in individuals aged 15-40 years. It has been mainly found in rural areas and seen in individuals bathing in pond or river. Patients with rhinosporidiosis of nose and nasopharyngeal airway mainly present with epistaxis and nasal obstruction. Respiratory distress or stridor have rarely been reported in these cases. Stridor is high pitched, noisy respiration produced by turbulent airflow through narrowed air passages. Stridor can be of 3 types- 1.Inspiratory, if the obstruction is in the pharynx or supraglottis. 2. Expiratory, if the obstruction is in the thoracic trachea or bronchii.3.Biphasic, if the obstruction is in the glottis, supraglottis or cervical trachea. Rhinosporidiosis can present with stridor in two manners- 1.Nasal or Nasopharyngeal rhinosporidiosis with hypopharyngeal and /or laryngeal extension 2. Laryngeal rhinosporidiosis. 


\section{Materials and Methods}

Our study consisted of 5 patients, of which 3 patients had nasal rhinosporidiosis with oropharyngeal and hypopharyngeal extension that eventually presented with stridor and 2 patients with laryngeal rhinosporidiosis. CT Scan of nose and paranasal sinuses, fibreoptic laryngoscope, histopathological examination were used to establish the diagnosis.

\section{Case Series}

Case 1: A 32 years old, male, rickshaw driver presented to the OPD with a pinkish polypoidal mass involving right nostril and sudden onset respiratory distress and stridor. He had history of recurrent epistaxis, nasal obstruction and foreign body sensation in throat earlier. On examination The mass was found to hang in the oropharynx. CT Scan of Nose and Paranasal sinuses with neck findings - Mass invoving right nasal cavity, extending into the nasopharynx, oropharynx and hypopharynx. Paranasal sinuses appear normal. No breach seen in the laryngeal cartilages. Other neck structures appear normal.

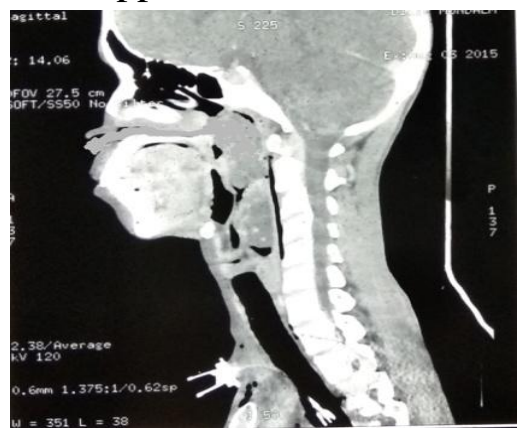

Fig1: Mass involving the right nasal cavity, extending into the oropharynx

Case 2: A 40 years old, female presented to the OPD with stridor, respiratory distress and a polypoidal mass involving the right nostril and a huge polypoidal mass occupying the oral cavity and the oropharynx. The nasal mass was present for about 2 months and the respiratory distress was gradually increasing in severity for last 5 days. There was no history of epistaxis present. CT Scan findings - Nasal and nasopharyngeal mass was found to extend into the oropharynx and hypopharynx.

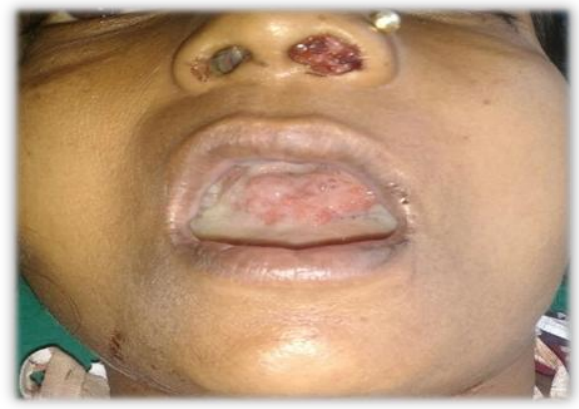

Fig 2: Polypoidal mass involving the left nasal cavity and oral cavity

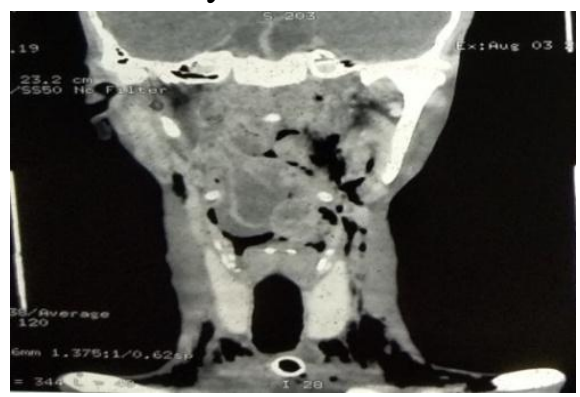

Fig 3: Mass involving the oropharynx and hypopharynx

Case 3: A 56 year old male presented to the ENT emergency with sudden onset respiratory distress and stridor. On examination: A polypoidal mass was found to hang in the oropharynx. Anterior rhinoscopy revealed a pinkish mass in bilateral nasal cavities. CT Scan findings: A homogenous mass was found to involve bilateral nasal cavities and extending into the oropharynx and hypopharynx.

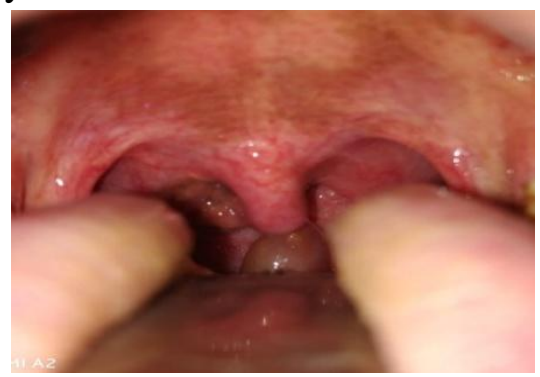

Fig 4: Mass hanging in the pharynx from the nose

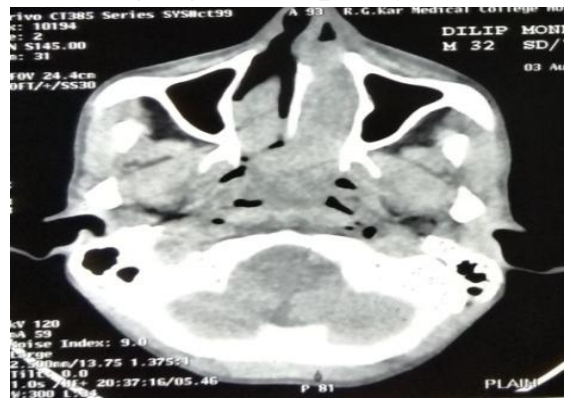

Fig 5: Mass involving both the nasal cavities extending into the pharynx 
Treatment - The treatment option was same for all the above 3 cases. Emergency Tracheostomy was performed. Patient was put on Nasal endoscopy was done to remove the nasal attachments and base cauterized. The mass was delivered perorally. Direct Laryngoscopy was done for further disease clearance. The excised mass was sent for Histopathological examination.

Case 4: A 32 years female presented with sudden onset respiratory distress with stridor .The patient had a previous history of nasal rhinosporidiosis which was excised and cauterized 6 months back. FOL findings- pinkish, friable mass involving the glottic chink. It was presumed to arise from the left vocal cord. CT Scan findings: An irregular mass was found to involve the glottic chink with subglottic extension.

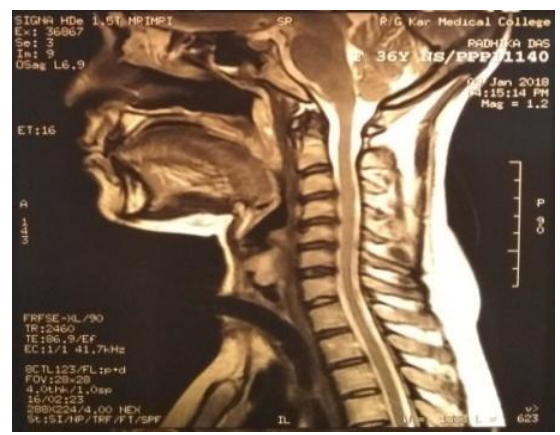

Fig 6: Mass involving the glottic chink with subglottic extension

Case 5: A 50 year old male presented stridor with respiratory distress for 3 days progressively increasing. He also had a history of nasal and oral rhinosporidiosis in the past. FOL findings: a pinkish friable mass was found to involve the left vocal cord with compromisation of the glottic chink. CT Scan findings: An irregular mass was found to involve the glottic chink.

Treatment: Tracheostomy was done to relieve stridor and the mass removed by LARYNGOFISSURE technique in both the above cases.

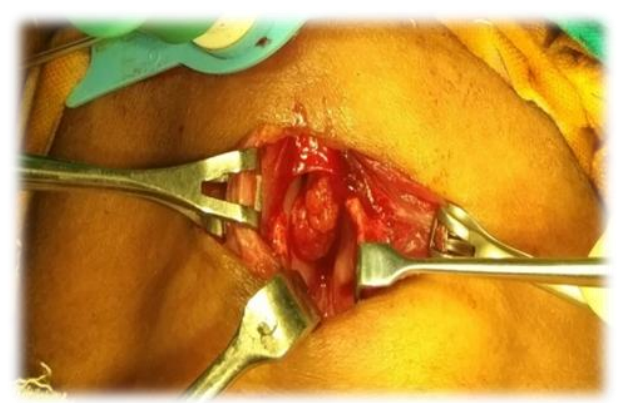

Fig 7: Laryngofissure technique showing mass involving the left vocal cord and occupying the glottic chink

\section{Discussion}

The first case of rhinosporidiosis was brought into light in the year 1892 by Malbran of Buenos Aires. The first detailed report on rhinosporidiosis was made by Seeber in the year 1900 . Herr et $\mathrm{al}^{3}$ classified the organism in a clade which was named the Mesomycetozoa, which includes fish and amphibian pathogens in the former DRIP clade (Dermocystidium, the rossette agent, Ichthyophonus and Psorospermium). Transmission to humans have been described by Demellow and Karunaratne. Karunaratne ${ }_{4}$ postulated that autoinoculation accounted for satellite lesions in the host tissue, which is widely accepted. Rhinosporidium seeberi, develops sporangia which contain endoconidia. The endoconidia are released from mature sporangia into the environment. The resistant conidia get implanted into the traumatic tissues of the susceptible hosts. Spillage of endospores from polyp after trauma or surgery is thought to be followed by autoinoculation into adjacent traumatic epithelium. The most common presentation of rhinosporidiosis is unilateral nasal obstruction or epistaxis with sessile or pedunculated soft friable and vascular polyps involving the nasal cavity. Other symptoms include rhinorrhea, postnasal drip with cough, coryza. Eye involvement is common. Stridor associated with rhinosporidiosis is a very rare presentation and there are only a few reports in history acknowledging it. In the year 2004, Kumar $S$ et al. reported a case of Laryngeal rhinosporidiosis, before which 3 such cases had 
been reported $_{5}$. Madana $\mathrm{J}$ et al. in the year 2010 reported a case of rhinosporidiosis of the upper airways and trachea 6 and Hansa Banjara et al. has presented a case of bronchial rhinosporidiosis 7 . Yet any case series with similar involvement has not been found to be reported in the history. Surgical excision is the treatment of choice. Surgical excision with cautery of base of lesion has shown to reduce the risk of recurrence 9. Dapsone (diaminodiphenylsulfone) has antirhinosporidial effect as it prevents maturation of sporangia and promotes fibrosis in the stroma. Hence, it has an important role in preventing recurrences. Chemotherapy has poor penetration of the sporangial wall of spherules and is not useful 8 . The use of KTP-532 laser for cauterization and vaporization on rhinosporidiosis mass $_{10}$ has shown to reduce the chances of seeding because of less physical contact with the tissues . In our study, the nasopharyngeal mass was removed endoscopically with cauterizatization of the bases and the oropharyngeal component was delivered perorally. Whereas, in the previous case reports on laryngeal rhinosporidiosis had the mass removed laryngoscopically, in our study we have employed laryngofissure technique for removal of the laryngeal mass, which is new application in such a scenario. All the patients were put on Dapsone, 100mg OD for 1 month and then every alternate day for next 5 months. Yet, 2 out of 5 patients had recurrence and had to undergo revision surgery while the dapsone treatment was continued. One amongst the two developed disseminated disease involving the skin of the neck arms and right leg along with the underlying muscle and is still continuing treatment with daps one.

\section{Conclusion}

Rhinosporidiosis is a quite prevalent in India and Srilanka, for the favourable climatic conditions and rampant tradition of pond bathing. Though, complete excision and electrocautery has become an acceptable means of treatment yet, the disease has no definitive treatment or management protocol. Recurrence is very common because of the propensity of the spores getting implanted onto traumatic adjacent tissues. Daps one has shown to have benefitted to the cause and prevent recurrence. Still, it's an area of research as to devise a proper curative treatment modality. Hematogenous spread of the disease though rare, can lead to disseminated disease involving viscera eventually and can be life threatening under adverse circumstances.

\section{References}

1. Kwon-Chung $\mathrm{Kj}$, Bennett JE. Rhinosporidiosis. In: Medical mycology. Philadelphia: Lea \& Febiger; 1992. P. 695706.

2. https://en.wikipedia.org/wiki/Rhinosporidi um_seeberi

3. Herr RA, Ajello L, Taylor JW, Arseculeratne SN, Mendoza L. Phylogenetic analysis of Rhinosporidium seeberi's 18S Small-subunit Ribosomal DNA groups this pathogen among members of the Protoctistan Mesomycetozoa clade. J clin microbiol 1999;37(9):2750-2754

4. Karunaratne WA. London: The Athlone Press; 1964. Rhinosporidiosis in Man. [Google Scholar]

5. Laryngeal rhinosporidiosis: report of a rare case.2004.Kumar $\mathrm{S}^{1}$, Mathew J, Cherian V, Rozario R, Kurien M.[PubMed]

6. Rhinosporidiosis of the upper airways and trachea.2010Madana $\mathbf{J}^{1}$, Yolmo D, Gopalakrishnan S, Saxena SK [PubMed]

7. Bronchial rhinosporidiosis: An unusual presentation. Hansa Banjara, Ravindra K. Panda, ${ }^{1}$ Ajit V. Daharwal, V. Sudarshan, ${ }^{2}$ Digvijay Singh, and Anuj Gupta. [PubMed]

8. Rhinosporidiosis: A Riddled Disease of Man and Animals Ruchi Tiwari 1, Kumaragurubaran Karthik2 , Kuldeep Dhama3, Muhammad Zubair Shabbir4, Sandip Kumar Khurana5 
9. Ezeanolue BC, Odike M. Nasal Rhinosporidiosis-A case report and outcome of therapy with Flucanozole. J Otorhinolaryngol Nig. 2004;1:22-24. [Google Scholar]

10. Kameshwaran M, Anand Kumar RS, Sathiya M, Raghunandhan S, Jacob J. KTP-532 Laser in the management of Rhinosporidiosis. IJOHNS. 2005;57:298300.[PMC free article] [PubMed] [Google Scholar]. 